\title{
Effects of Round Window Opening Size and Moisturized Electrodes on Intracochlear Pressure Related to the Insertion of a Cochlear Implant Electrode
}

\author{
Ingo Todt Arneborg Ernst Philipp Mittmann \\ Department of Otolaryngology, Head and Neck Surgery, Unfallkrankenhaus Berlin, \\ Berlin, Germany
}

\section{Key Words}

Hearing preservation - Intracochlear pressure $\cdot$ Round window · Moisturized cochlear implant electrode

\begin{abstract}
Intracochlear pressure changes during the cochlear implant insertion are assumed to be an important contributor to hearing preservation. The aim was to observe intracochlear pressure changes by different round window opening sizes and different hydrophilic electrode conditions. The experiments were performed in a cochlear model with a micropressure sensor in the helicotrema area. Different artificial round window membrane and different moisturized electrode conditions were compared. A punctured round window causes a significantly higher and an indirect moisturized electrode condition a significantly lower intracochlear pressure change. The degree of round window opening and the hydrophilic character of an electrode during insertion affect the intracochlear pressure significantly in a model.
\end{abstract}

(C) 2016 The Author(s)

Published by S. Karger AG, Basel

\section{Introduction}

With expanded indication criteria for cochlear implantation, patients with residual hearing have become a growing share of cochlear implant (CI) candidates. Those patients address the question of hearing preservation during surgery, which is reasonable to achieve a best possible clinical and audiological benefit from the $\mathrm{CI}$, and the possibility of fitting an EAS/hybrid system. Several factors are discussed contributors to the goal of preserved 


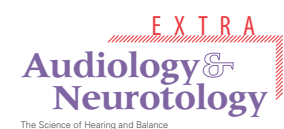

Fig. 1. Photograph of a FOP-MA sensor.

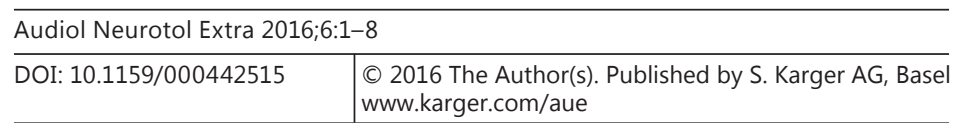

Todt et al.: Effects of Round Window Opening Size and Moisturized Electrodes on Intracochlear Pressure Related to the Insertion of a Cochlear Implant Electrode

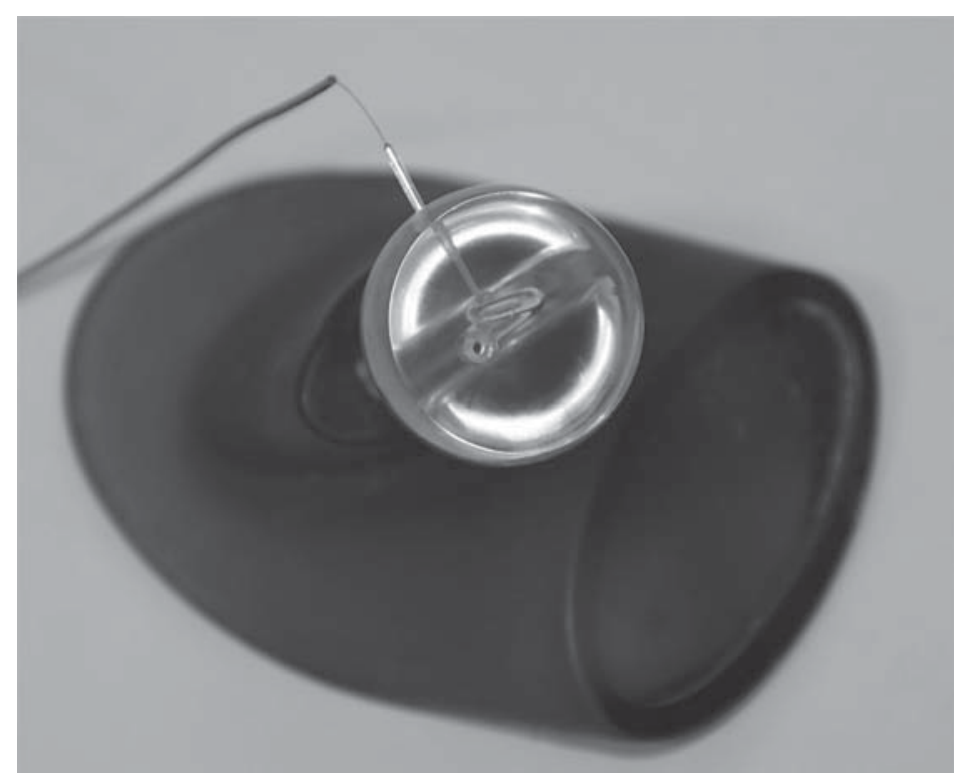

residual hearing, i.e. the design of atraumatic electrodes, modifications of 'soft surgery' techniques, by considering insertional forces [Kontorinis et al., 2011] and intracochlear fluid pressure (ICFP) changes [Roland et al., 2007]. Intracochlear pressure changes were shown to directly correlate with the insertional speed of the electrode in a cochlear model [Todt et al., 2014]. Observations of the role of the round window in an insertion concept are limited. Different forms of opening an artificial round window have been shown to cause significant differences in ICFP changes [Mittmann et al., 2014].

Since the insertion of a CI electrode leads to a fluid volume displacement in the cochlea, the question of where this fluid volume can surpass occurs. The physiological main pressure equilibration pathway of the cochlea, the aquaeductus cochleae, is an unreliable pressure equilibrator since its patency is highly variable [Gopen et al., 1997]. Therefore, the extent of round window opening should also be considered as equilibration route for intracochlear perilymph displaced by the electrode. Additionally, the impact of electrode surface-changing additives on insertion forces has been described in terms of a lubricant function [Kontorinis et al., 2011]. The occurrence of surface tension forces between the hydrophobic silicone electrode and the intracochlear fluid has not yet been observed. It was therefore our aim to investigate the influence of different round window openings and electrode moisturizing on intracochlear pressure changes in a model cochlea.

\section{Material and Methods}

\section{Pressure Sensor}

Intracochlear pressure was measured using the micro-optical pressure sensor developed by Olson [1998]. Sensors are made by FISO (Quebec, Canada); the used model is an 8-mm FOP-MA (fig. 1). Basically, the tip of the pressure sensor was a hollow glass tube sealed on one end by a thin plastic film diaphragm coated with a reflective surface of evaporated gold. The optical fibre was mounted on the glass tube at a distance (50-100 $\mu \mathrm{m})$ to the diaphragm tip. The optical fibre was attached to an LED light source and a photodiode sensor. Light from the LED source reached the sensor tip of the optical fibre, fanned out as it left the fibre and was 


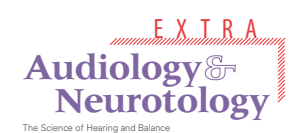

Audiology

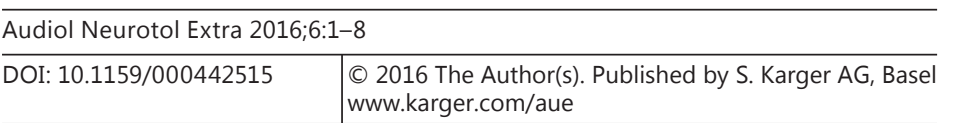

Todt et al.: Effects of Round Window Opening Size and Moisturized Electrodes on Intracochlear Pressure Related to the Insertion of a Cochlear Implant Electrode

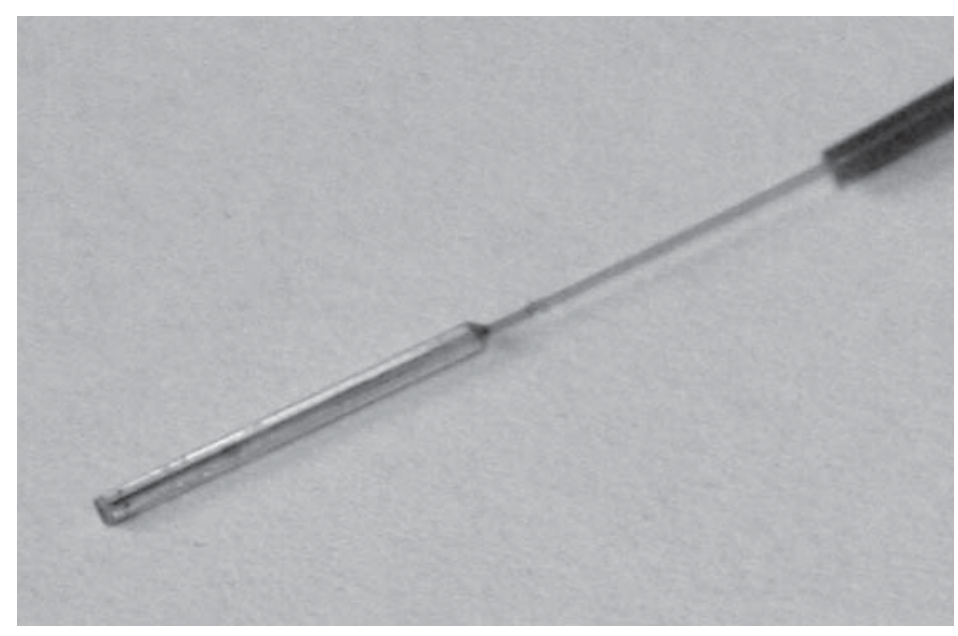

Fig. 2. Photograph of the used full scalar model.

reflected by the gold-covered flexible diaphragm. The reflected light was sensed by the photodiode, and light pressure-induced distance displacements of the diaphragm modulated the intensity of the reflected light. The sensor was connected to a module which was again linked to a PC. Evolution software was used to record intracochlear pressure. Resolution for the specific sensor (FOP-MA)/controller (FPI-HS) combination is $0.01 \mathrm{~mm} \mathrm{Hg}$. Sensitivity for the used sensor is $-3.12 \mathrm{~nm} / \mathrm{mm} \mathrm{Hg}$ in the used range. Time resolution is 5,000 Hz. Calibration was repeatedly performed against atmospheric pressure. One mm $\mathrm{Hg}$ amounts to $133.3 \mathrm{~Pa}$ (MKS unit).

\section{Model Cochlea}

The model was a full scalar model of the cochlea with a volume of $87 \mathrm{~mm}^{3}$, which is slightly above the physiological range [Kirk and Gosselin-Ildari, 2009] (fig. 2). The sensor was positioned through a drilled hole in the apical region of the cochlea, fixed in its position by fibrin glue and placed within the channel in such a way that the tip was not in contact with the edge of the channel or the ground. Afterwards, the cochlea was filled with sterile water and microscopically controlled to exclude any enclosed air bubbles. The experiments were performed in series with a sensor in an unchanged position to exclude a sensor positionrelated bias and allow an interexperimental analysis of the series.

\section{Experiments}

Experiments with Differently Sized Round Window Openings

Insertions through the round window opening $(1.5 \mathrm{~mm})$ were performed with three differently sized openings. Thin polyethylene foil simulated the round window membrane. In the first opening condition, there was no foil (fig. 3). In the second condition, the foil covered half of the round window opening (fig. 4). In the third condition, a needle punched the foil and this opening was positioned over the round window opening (fig. 5). All experiments were performed with a HiFocus IJ electrode using an insertion tool. Insertion speed was $50 \mathrm{~s}$ to $1 \mathrm{~min}(0.3-0.36 \mathrm{~mm} / \mathrm{s})$. All experiments were performed with five repetitions.

Surface Tension Experiments

Dry Insertion. Dry electrodes were inserted in the artificial cochlea without a round window membrane. 
Audiology
Neurotology

Fig. 3. Full opening of the round window.

Fig. 4. Half-opened round window.

\begin{tabular}{l|l}
\hline Audiol Neurotol Extra 2016:6:1-8 \\
\hline DOI: 10.1159/000442515 & $\begin{array}{l}\text { (c) 2016 The Author(s). Published by S. Karger AG, Basel } \\
\text { www.karger.com/aue }\end{array}$ \\
\hline
\end{tabular}

Todt et al.: Effects of Round Window Opening Size and Moisturized Electrodes on Intracochlear Pressure Related to the Insertion of a Cochlear Implant Electrode
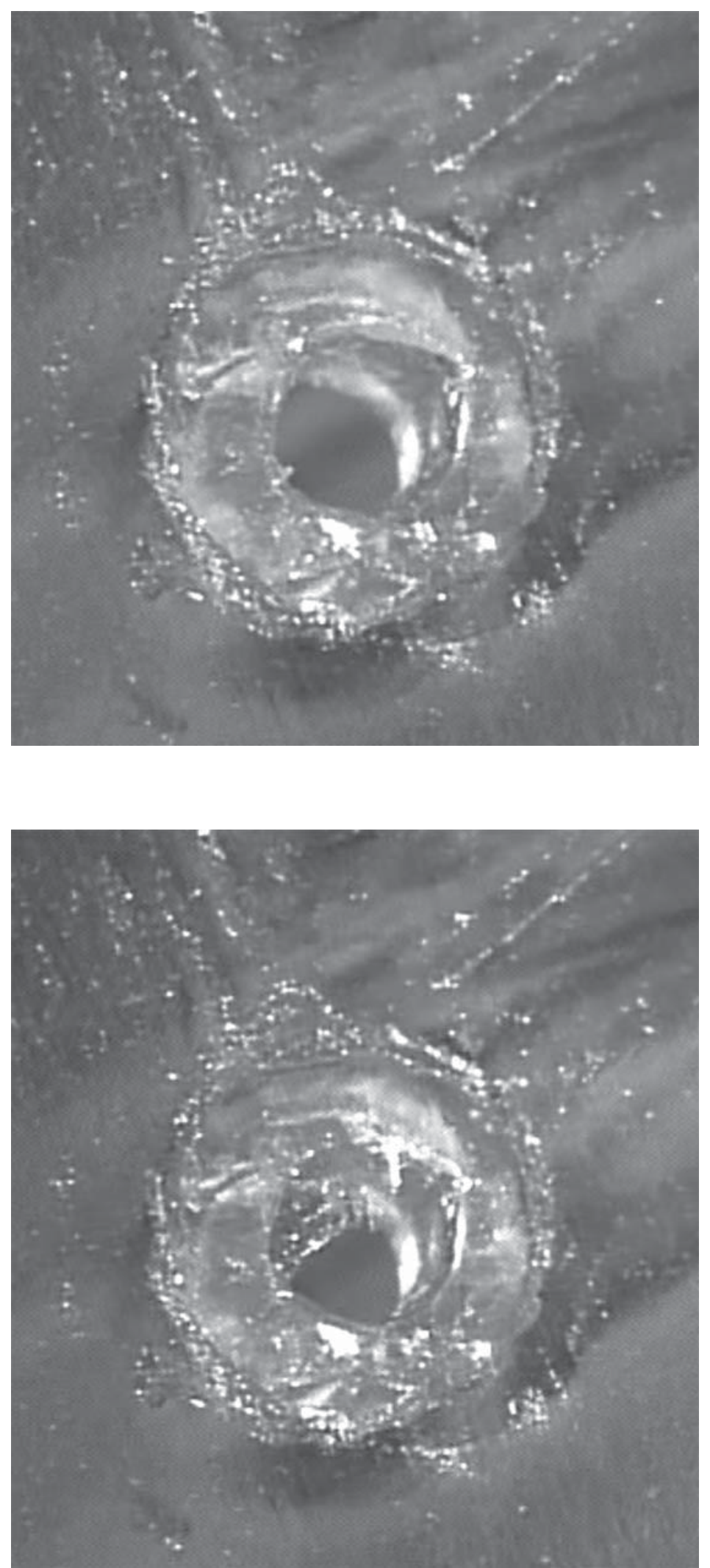


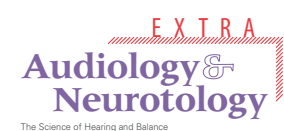

Fig. 5. Punctured round window.

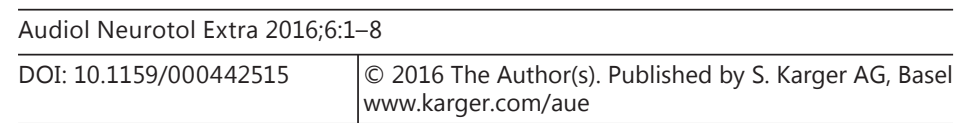

Todt et al.: Effects of Round Window Opening Size and Moisturized Electrodes on Intracochlear Pressure Related to the Insertion of a Cochlear Implant Electrode

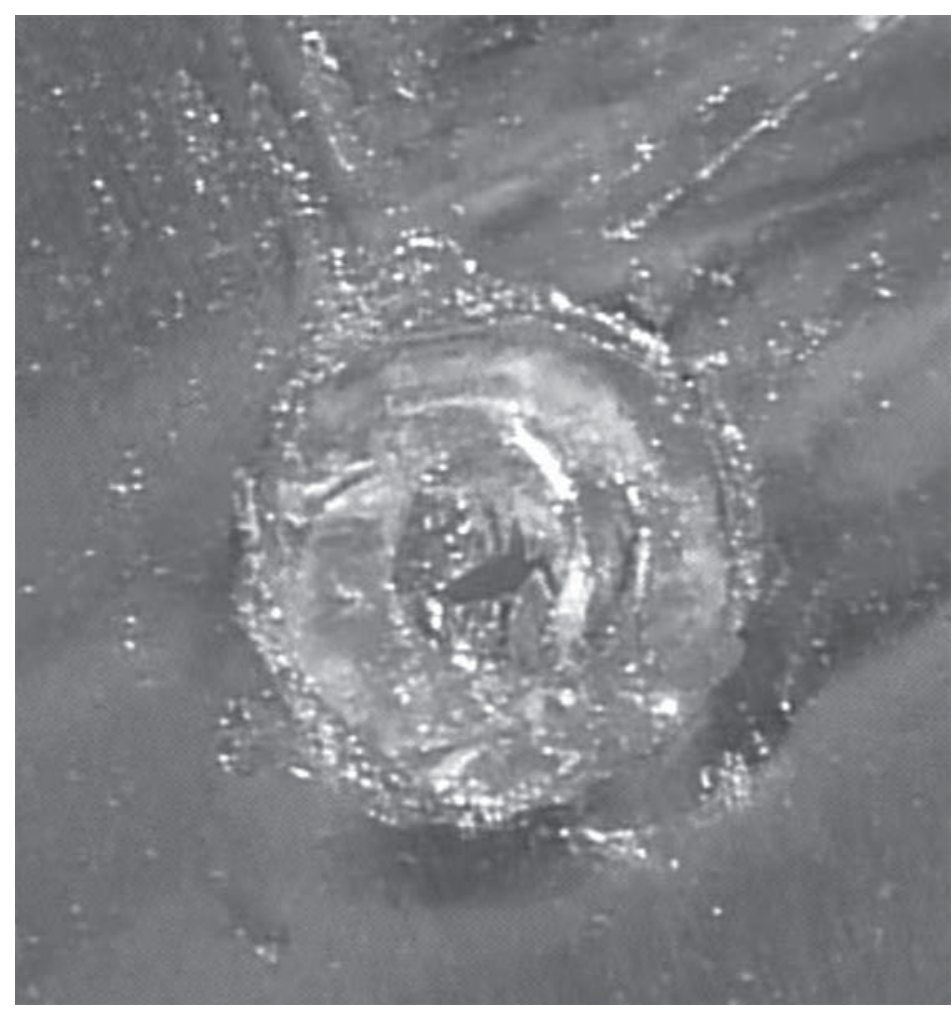

Directly Moisturized Insertion. Electrodes were inserted after bathing them in water and holding a drop on the tip of the electrode during insertion.

'Overfilled' Moisturized Insertion. Electrodes were inserted through a round window which was overfilled with water (surface tension was significant in a separate water drop outside the round window plane).

Indirect Moisturized Insertion. Electrodes were inserted by placing the electrode tip into some water beneath the artificial round window. Then, the water-bathed tip of the electrode was brought into the artificial round window and inserted without losing water contact.

\section{Results}

A one-way ANOVA was conducted to determine if the ICFP changes were different for variable round window sizes. There were no outliers in the data, as assessed by box plotting. Maximum ICFP changes were normally distributed for all insertion techniques applied, as assessed by Shapiro-Wilk's test ( $p>0.05$ ). There was homogenicity of variances, as assessed by Levene's test for equality of variances $(p=0.113)$. Data are presented in mm $\mathrm{Hg}$ (mean \pm SD). The ability to reduce the maximum ICFP changes was statistically significantly different for variable insertion techniques $[\mathrm{F}(2,6)=17.689, \mathrm{p}=0.003]$. Maximum amplitude changes were increased from half-opened round window $(0.47 \pm 0.06)$ to fully opened $(0.48 \pm 33)$ to punctured round window $(1.83 \pm 0.45)$. Tukey's post-hoc analysis revealed that the increase was significant for both the punctured opening versus the half opening and the punctured opening versus the fully opened round window (fig. 6).

A one-way ANOVA was conducted to determine if the maximum ICFP changes were different for variable moisture conditions. There were no outliers in the data, as assessed by 
Fig. 6. Exemplaric intracochlear pressure behaviour during a CI insertion with various opening sizes of the round window.

\section{\begin{tabular}{l|l}
\hline DOI: $10.1159 / 000442515$ & C 2016 The Author(s). Published by S. Karger AG, Basel
\end{tabular} www.karger.com/aue}

Todt et al.: Effects of Round Window Opening Size and Moisturized Electrodes on Intracochlear Pressure Related to the Insertion of a Cochlear Implant Electrode

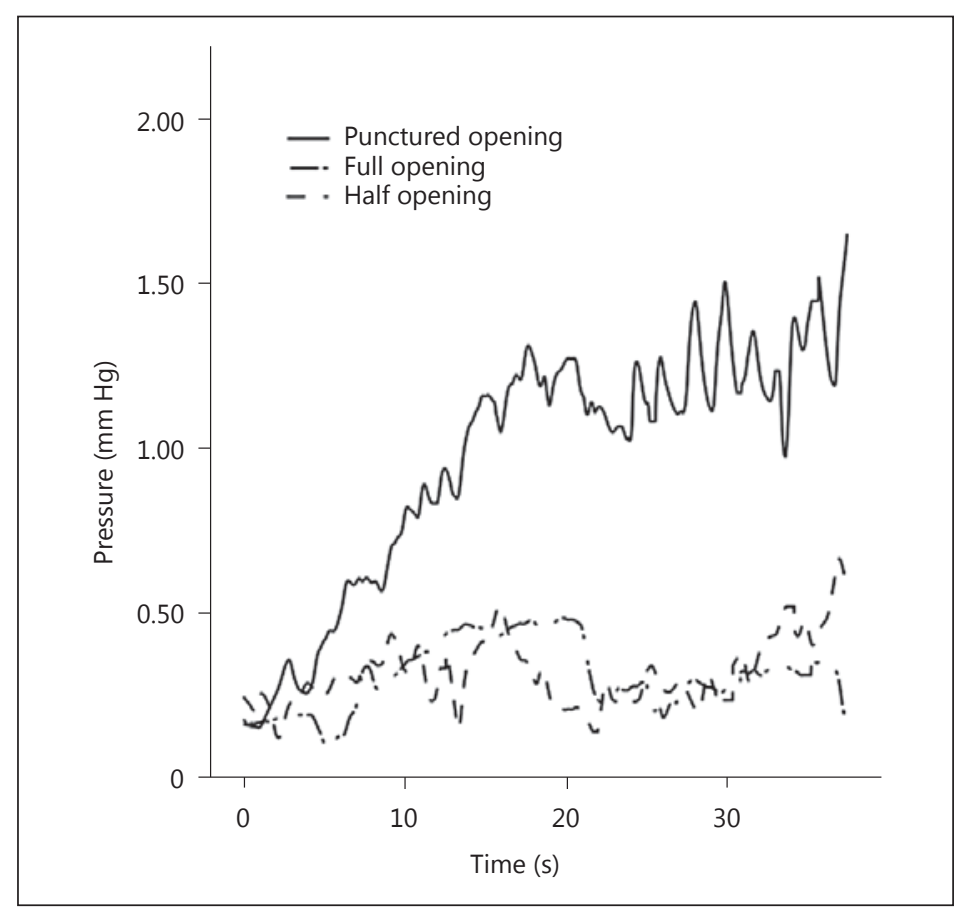

box plotting. Maximum ICFP changes were normally distributed for all qualities, as assessed by Shapiro-Wilk's test $(\mathrm{p}>0.05)$. There was homogenicity of variances, as assessed by Levene's test for equality of variances $(p=0.738)$. Data are presented in $\mathrm{mm} \mathrm{Hg}$ as mean \pm $\mathrm{SD}$. The ability to reduce the maximum ICFP changes was statistically significantly different for variable moisture characteristics $[F(3,12)=53.004, p<0.0005]$. Maximum ICFP changes were decreased from dry electrode $(0.63 \pm 0.05)$ to moisturized tip $(0.35 \pm 0.1)$ to overfilled moisturized insertion $(0.11 \pm 0.05)$ to indirectly moisturized insertion $(0.1 \pm 0.07)$. Tukey's post-hoc analysis revealed that the decrease was significant for several moisture characteristics (fig. 7).

\section{Discussion}

The physiological hearing is characterized by a translation of acoustic energy (dB SPL) into mechanical energy that drives the ossicular chain $(\mathrm{mN})$, finally leading to a hydromechanical pressure change intracochlearly (mm Hg, psi, Pascal). Excessive, pathophysiological acoustic energy levels are therefore assumed to lead to high static intracochlear pressure changes or fast pressure changes with a high angular speed [Bohmer, 1993; Dancer and Franke, 1980]. This phenomenon underlies the hypothesis that insertional forces induced by the CI electrode influence the ICFP and thus, have an impact on residual hearing.

The insertion of CI electrodes has been shown to lead to a speed-dependent intracochlear pressure increase in a cochlear model [Todt et al., 2014] with a static pattern of pressure change. In addition to this finding, the way of opening the round window has been shown to cause specific pressure changes inside the cochlea [Mittmann et al., 2014], with a fast pattern of pressure gradients.

The insertion-dependent increase of fluid pressure raised the question of natural pathways of pressure equilibration, e.g. the cochlear aqueduct, the endolymphatic sac, the 
Fig. 7. Exemplaric intracochlear pressure behaviour during a CI insertion and different moisturized conditions.

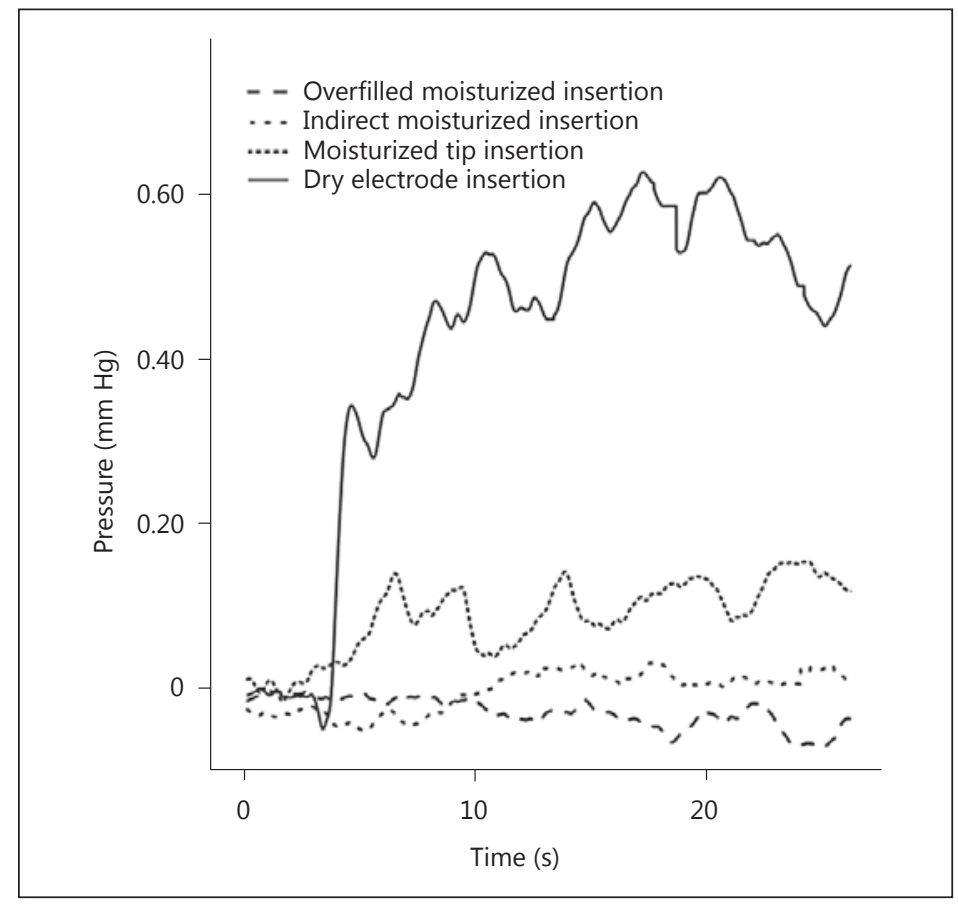

vestibular aqueduct and the modiolus. While the latter three are known to be slowly reacting pressure equilibrators [Ciuman, 2009], the fast-reacting cochlear aqueduct is highly variable in its patency. It was described to be open in about one third of temporal bones [Gopen et al., 1997], and this patency is discussed to be age-dependent [Wlodyka, 1978]. In turn, this variation might have clinical consequences, such as the higher rate of hearing preservation in younger patients [Anagiotos et al., 2015], leaving the cochlear aqueduct not ideally as a pressure equilibrator for the whole patient population. Therefore, the size of the round window opening seems to be crucial for intracochlear pressure gradients during the array insertion.

So far, most surgeries for hearing preservation performed a slit or puncture opening of the round window. Our results (fig. 6) show that this technique leads to significantly higher intracochlear pressure gradients than did an insertion in a half-open or wide-open round window in our model. Based on this modelling approach, a puncture or a slit opening cannot be recommended anymore under the aspect of preventing the generation of large intracochlear pressure gradients. It remains to be elucidated whether the above favoured round window openings have repercussions on the final sealing of the cochlea.

Electrode surfacing was observed so far either to decrease insertion forces by lubricants applied [Kontorinis et al., 2011] or to carry neuroprotective substances into the cochlea via the electrode array [Stathopoulos et al., 2014]. In our model experiments, the contact of a hydrophobic silicon electrode array with the intracochlear fluid led to measureable pressure changes inside the cochlea. We therefore performed four different methods of insertion and found an indirect technique of overfilling the round window niche with water to be most effective than directly bathing the electrode before insertion (fig. 7). This difference might rely on a more effective hydrophilisation of the electrode tip before entering the cochlea. An out-of-the-cochlea hydrophilisation might lead to smaller polarised shields that displaced the intracochlear fluid. This experimental effect should be confirmed in a clinical setup. 


\section{Conclusion}

The degree of round window opening and the hydrophilic character of the CI electrode surface affect the intracochlear pressure changes during the electrode insertion. A wide opening of the round window and a hydrophilic condition of the electrode are recommended based on our experimental results.

\section{Acknowledgement}

This study was supported by Advanced Bionics, Stäfa, Switzerland.

\section{References}

Anagiotos A, Hamdan N, Lang-Roth R, Gostian AO, Luers JC, Huttenbrink KB, Beutner D: Young age is a positive prognostic factor for residual hearing preservation in conventional cochlear implantation. Otol Neurotol 2015;36:28-33.

Bohmer A: Hydrostatic pressure in the inner ear fluid compartments and its effects on inner ear function. Acta Otolaryngol Suppl 1993;507:3-24.

Ciuman RR: Communication routes between intracranial spaces and inner ear: function, pathophysiologic importance and relations with inner ear diseases. Am J Otolaryngol 2009;30:193-202.

Dancer A, Franke R: Intracochlear sound pressure measurements in guinea pigs. Hear Res 1980;2:191-205.

Gopen Q, Rosowski JJ, Merchant SN: Anatomy of the normal human cochlear aqueduct with functional implications. Hear Res 1997;107:9-22.

Kontorinis G, Paasche G, Lenarz T, Stover T: The effect of different lubricants on cochlear implant electrode insertion forces. Otol Neurotol 2011;32:1050-1056.

Kirk EC, Gosselin-Ildari AD: Cochlear labyrinth volume and hearing abilities in primates. Anat Rec 2009;292:765776.

Mittmann P, Ernst A, Todt I: Intracochlear pressure changes due to round window opening: a model experiment. ScientificWorldJournal 2014;2014:341075.

Olson ES: Observing middle and inner ear mechanics with novel intracochlear pressure sensors. J Acoust Soc Am 1998; 103:3445-3463.

Roland PS, Wright CG, Isaacson B: Cochlear implant electrode insertion: the round window revisited. Laryngoscope 2007;117:1397-1402.

Stathopoulos D, Chambers S, Enke YL, Timbol G, Risi F, Miller C, Cowan R, Newbold C: Development of a safe dexamethasone-eluting electrode array for cochlear implantation. Cochlear Implants Int 2014;15:254-263.

Todt I, Mittmann P, Ernst A: Intracochlear fluid pressure changes related to the insertional speed of a CI electrode. Biomed Res Int 2014;2014:507241.

Wlodyka J: Studies on cochlear aqueduct patency. Ann Otol Rhinol Laryngol 1978;87:22-28. 